\title{
Effects of timing of pre-exercise nutrient intake on glucose responses and intermittent cycling performance
}

\author{
Kelly Pritchett (PhD, RD) ${ }^{1,2}$ \\ Philip Bishop (EdD) ${ }^{1}$ \\ Robert Pritchett (PhD) ${ }^{2}$ \\ Mark Kovacs (PhD) ${ }^{1}$ \\ JK Davis (MS) ${ }^{1}$ \\ Catalina Casaru ${ }^{1}$ \\ Matt Green (PhD, FACSM) ${ }^{3}$ \\ ${ }^{1}$ Kinesiology Department, University of Alabama, Tuscaloosa, AL \\ ${ }^{2}$ Department of Health, Human Performance, and Nutrition, Central Washington University, Ellensburg, WA \\ ${ }^{3}$ Department of Health, Physical Education, and Recreation, The University of North Alabama. Florence, AL
}

\begin{abstract}
Objective. To evaluate the effects of two different pre-exercise feeding schedules ( 15 minutes and 60 minutes prior to exercise) of a mixed-nutrient nutritional bar on blood glucose levels and subsequent intermittent, high-intensity cycling performance.

Methods. Ten moderately trained athletes participated in this counterbalanced, crossover, repeated measures study. Participants completed a 50-minute counterbalanced treatment intermittent exercise protocol. During one trial, participants consumed $400 \mathrm{ml}$ water and a nutritional bar 15 minutes before the exercise session (15MPE). During another trial, participants consumed $400 \mathrm{ml}$ water and a nutritional bar 60 minutes before the exercise session (60MPE). During a control trial (CON) participants consumed $400 \mathrm{ml}$ water.
\end{abstract}

Results. There were no significant differences in plasma glucose response at rest or during exercise among the three treatments (CON, 15MPE and 60MPE). There were no significant differences in mean power (MP) between the three trials.

Conclusions. Pre-exercise nutrient feedings at 15 minutes or 60 minutes before exercise did not affect intermittent cycling performance or blood glucose concentration. These results suggest that the time of ingestion, within 1 hour prior to exercise, of a complex carbohydrate similar in composition and volume used in this study does not impact on performance.

\section{CORRESPONDENCE:}

Kelly Pritchett

Department of Health, Human Performance, and Nutrition

Central Washington University

Ellensburg, WA 98926

205-887-1809

E-mail: Kkerr@cwu.edu

\section{Introduction}

The position statements of the American College of Sports Medicine (ACSM) and the American Dietetic Association (ADA) have concluded that the recommendations on carbohydrate consumption within 1 hour prior to activity are questionable. ${ }^{1}$ It is well known that carbohydrate $(\mathrm{CHO})$ ingestion during exercise can enhance performance when the exercise duration is longer than 45 minutes. ${ }^{7}$ Research on the impact of pre-exercise nutrition on performance, especially within an hour prior to exercise, remains controversial. ${ }^{14}$ Studies have shown increases, ${ }^{11,17,22,24,25}$ no effect, ${ }^{4,7,8,13,23}$ or even decreases in performance ${ }^{10,14}$ after $\mathrm{CHO}$ ingestion within the hour prior to exercise. More recently, researchers have begun manipulating the timing of pre-exercise nutrition to determine the effects on subsequent exercise performance. $^{19}$

The majority of pre-exercise nutritional studies have examined carbohydrate-only administration in liquid form, and its subsequent effect on steady-state endurance cycling performance. ${ }^{12,14}$ However, a liquid carbohydrate drink may not be representative of all pre-exercise nutritional practices, since many athletes may choose to consume a solid meal or energy bar and not necessarily a liquid carbohydrate drink. Many of these energy bars are marketed as nutritional fuel support for exercise, and are accompanied by suggestions for consumption before, during, and after exercise. Furthermore, most commercial nutritional bars and supplements being used by athletes consist of a blend of different percentages of the macronutrients, yet little research has examined the effects of a blend of the macronutrients (carbohydrate, protein, fat) typical of most nutritional bars on exercise performance. ${ }^{20}$ Controversy exists over the optimal composition and quantity of macronutrients as well as the optimal timing of nutrient intake in the hour prior to exercise. $^{14,20}$

Little research has looked at mixed-nutrient nutritional bars and the timing of their ingestion before intermittent high-intensity exercise. Therefore, it would be inappropriate to generalise the results seen in steady-state cycling to apply to intermittent sporting events as well. Examining high-intensity intermittent exercise would be more repre- 
sentative of many sporting events (i.e. soccer, basketball and rugby). Pre-exercise recommendations suggest ingesting $1 \mathrm{~g} \mathrm{CHO} / \mathrm{kg}$ body weight if an athlete chooses to eat 1 hour prior to exercise. ${ }^{5}$ However, recommendations regarding pre-exercise meal ingestion within the time period of less than 1 hour prior to exercise are not currently based on sufficient research. ${ }^{1}$

In most athletes, at the onset of exercise, we expect a rise in circulating insulin, which increases muscle glucose uptake. When a meal is ingested prior to exercise the synergistic combination of the insulinaemic effect of exercise and the insulin released to counteract the pre-exercise meal may result in rebound hypoglycaemia after $\sim 30$ minutes of exercise. 'Rebound' or 'reactive' hypoglycaemia is a term used to describe this fast rise and then sudden drop in glucose levels during exercise, when carbohydrates are ingested within the 60 -minute time period prior to exercise. ${ }^{14,15}$ Although this phenomenon has been consistently reported in the literature, $4,12,14,15$ it has not been shown to reduce performance in steady-state endurance exercise. $^{12}$

The purpose of this study was to investigate the effects of preexercise nutrient timing ( 15 and 60 minutes prior to exercise) of a mixed-nutrient nutritional bar (160 calories, $20 \mathrm{~g}$ carbohydrate, $11 \mathrm{~g}$ protein, and $4.5 \mathrm{~g}$ fat) on blood glucose response and subsequent intermittent high-intensity exercise performance. Perhaps the exercise intensity used in previous studies that investigated pre-exercise nutrition was not intense enough (i.e. did not require a high enough percentage of fuel from carbohydrate) to be affected by a decrease in blood glucose.

We hypothesised that the 15-minute pre-exercise feeding would result in a drop in blood glucose during the first 30 minutes of exercise, that the 60-minute pre-feeding would yield a favourable plateau in blood glucose, ${ }^{1}$ and that the performance following the 15-minute ingestion would be lower than that for the 60 -minute ingestion trial or the control condition.

\section{Methods}

\section{Subjects}

Ten healthy, moderately trained (more than 8 hours of training per week) male cyclists and triathletes were recruited to participate in this counterbalanced, crossover repeated-measures study. All subjects participated on a voluntary basis. Their descriptive characteristics are presented in Table I. Upon arrival, subjects were fully informed of the purposes and risks associated with the study procedures and written informed consent was obtained. Based on an alpha level of 0.05 , a beta value of 0.80 and a standard deviation of 1.0 for glucose values seen in pilot data, an a priori power analysis indicated a need for 10 subjects. All the procedures for this study were approved by the University of Alabama IRB. Each participant arrived at the laboratory after an 8-hour fast, and having abstained from caffeine and alcohol for a minimum of 24 hours.

\section{$\mathrm{VO}_{2 \max }$ and experimental protocol}

Each participant performed an introductory testing and familiarisation session, and subsequently 3 separate experimental trials (for a total of 4 sessions). $\mathrm{VO}_{2 \max }$ was determined during incremental cycling exercise to volitional fatigue on a cycle ergometer (Monark 515 Varberg, Sweden). Seat height was appropriately set for each

\section{TABLE I. Descriptive characteristics (mean $\pm \mathrm{SD} ; \mathrm{N}=10$ )}

\begin{tabular}{ll}
\hline Age (years) & $23.4 \pm 4.4$ \\
Height $(\mathrm{cm})$ & $180.6 \pm 5.5$ \\
Weight $(\mathrm{kg})$ & $77.1 \pm 17.7$ \\
Body fat $(\%)$ & $12.1 \pm 2.6$ \\
$\mathrm{VO}_{2 \mathrm{max}}\left(\mathrm{ml} \mathrm{kg}^{-1} \mathrm{~min}^{-1}\right)$ & $64.8 \pm 6.3$ \\
Peak work load $(\mathrm{kp})$ & $6.0 \pm 0.3$ \\
\hline
\end{tabular}

individual, with handlebars adjusted based on individual preference. Participants were required to maintain a cadence of $60 \mathrm{rev} \cdot \mathrm{min}^{-1}$ by using a metronome (Franz Country Technology, Gays Mills, WI, USA). Participants were fitted with an appropriately sized air-cushioned facemask (Vacu-med, Ventura, CA, USA), and a heart rate (HR) monitor transmitter (Polar, Stamford, CN, USA) at the level of the sternum. Expired air was directed through a valve (Hans Rudolf, $\mathrm{KC}, \mathrm{MO}$ ) and plastic tubing connected to a metabolic cart (Vacumed, Ventura, CA, USA), which was calibrated prior to each test with a known gas composition. A 7-L syringe (Hans Rudolph, Kansas City, MO, USA) was used to calibrate the system for measurement of ventilation. Participants completed a 2-minute warm-up on the cycle ergometer with no resistance to the flywheel. The workload was then increased by $0.5 \mathrm{kp}$ every minute thereafter.

The following criteria for terminating an exercise test were followed, based on ACSM guidelines: ${ }^{2}$

- the participant requested that the test be stopped for any reason

- the participant reached volitional exhaustion

- the participant displayed signs or symptoms which indicate the exercise test should be stopped (poor perfusion, ataxia, pallor, etc.)

- the participant could no longer maintain the required workload during testing

- the testers felt for any reason it was unsafe for the participant to continue.

Criteria for achievement of $\mathrm{VO}_{2 \max }$ were:

- rated perceived exertion $(\mathrm{RPE})>18$ (Borg 6 - 20 scale)

- respiratory exchange ratio $(\mathrm{RER})>1.1$

- plateau of $\mathrm{VO}_{2}$ with increased workload

- $85 \%$ of age-predicted maximum HR.

Two or more of these criteria were met by all participants. ${ }^{18}$

Participants, on three subsequent occasions separated by a washout period of 48 hours, completed a 50-minute (similar to many intermittent sporting events) counterbalanced exercise protocol designed to simulate intermittent sporting activities such as soccer, volleyball, football, tennis, basketball, etc. The protocol was performed on 3 separate days with at least 48 hours, but no longer than 1 week, between trials. On each occasion, the participant performed three identical exercise sessions (described below), preceded by one of three different nutritional strategies on each trial. Participants consumed a commercially available, low-GI nutritional bar (USANA, Salt Lake City, UT) containing 160 calories, $4.5 \mathrm{~g}$ fat ( $2 \mathrm{~g}$ saturated fat), $20 \mathrm{~g}$ carbohydrate ( $2 \mathrm{~g}$ dietary fibre) and $11 \mathrm{~g}$ protein along with 400 $\mathrm{ml}$ water to ensure adequate hydration prior to activity. For two treatments, participants were provided with the nutritional bar (USANA, Salt Lake City, UT) for each of the trials, but given at different times 
prior to exercise (15 minutes or 60 minutes prior to exercise). Participants completed a 5-minute cycling warm-up on the ergometer at $60 \%$ of $\mathrm{VO}_{2 \max }$ (which was specific to each person's fitness level and has been shown in many previous studies to allow the person to exercise at constant 'steady-state' where physiological variables (heart rate and oxygen requirements) should remain stable. After completing the warm-up session, participants completed $3 \times 10$-second 'allout' bicycle modified Wingate sprints interspersed with 50 -second rest periods. This 8-minute cycling period was repeated a total of 6 times (18 total modified Wingate tests). $\mathrm{RPE}^{3}$ measurements and HR were recorded following each Wingate sprint.

After the third Wingate sprint, blood was immediately collected during the rest period. The same procedures (5-minute cycling at $60 \%$ of $\mathrm{VO}_{2 \max }$, followed by $3 \times 10$-second modified Wingate sprints) were repeated a total of 6 times. During one trial, participants consumed $400 \mathrm{ml}$ water and a standardised nutritional bar 15 minutes before the exercise session (15MPE). During another trial, participants consumed $400 \mathrm{ml}$ water and a standardised nutritional bar 60 minutes before the exercise session (60MPE). In a control trial (CON) participants consumed $400 \mathrm{ml}$ water immediately prior to exercise. Participants then sat quietly until the commencement of the exercise session. Subjects were permitted to read or watch television between the ingestion of food and exercise time.

\section{Measurements}

Age $(\mathrm{y})$, height $(\mathrm{cm})$ and mass $(\mathrm{kg})$ were recorded with body fat percentage estimated using Lange skinfold calipers (Cambridge, Md, USA) and a 3-site method (chest, abdomen, thigh). ${ }^{19}$ A pre-trial (PRE) blood sample was collected at the fingertip using a capillary tube (Analox Inc., Boston, MA, USA) before the beginning of each trial to determine baseline (resting) blood glucose concentration. After subjects were given the treatment, a capillary blood sample was taken again at the onset of exercise $(0 \mathrm{~min})$, and after the third Wingate sprint at $8,16,24,32,40$, and 48 minutes using a capillary tube (Analox Inc., Boston, MA, USA). Blood samples were analysed for blood glucose concentration using an automated analyser (ANALOX PGM-7, Analox Inc., Boston, MA, USA). To improve reliability, each sample was analysed $\mathrm{mmol} \cdot \mathrm{I}^{-1}$ at least in duplicate with serial samples no greater than $0.2 \mathrm{mmol} \cdot \mathrm{I}^{-1}$ apart. The average of the two samples was used for analysis. The analyser was calibrated prior to each trial using a $5 \mathrm{mmol}$ and $8 \mathrm{mmol}$ glucose standard. For the purpose of this study, a glucose concentration $<3.5 \mathrm{mmol} \cdot \mathrm{I}^{-1}$ was classified as hypoglycaemia. ${ }^{9}$

During the trials, heart rate was recorded every minute (at $45 \mathrm{sec}-$ onds during the steady state and immediately after each 10 -second Wingate test). Every minute during each of the trials, subjects rated their perceived exertion (RPE) using a 6 - 20 Borg category scale. ${ }^{17}$ RPE was recorded for legs (RPE-L), chest (RPE-C), and overall (RPE-0). During each Wingate sprint cycle, the peak power (PP), mean power (MP) and percentage decrease were recorded using computer software (SMI St Cloud Minnesota, USA) integrated with the ergometer.

\section{Data analysis}

Basic descriptive characteristics were computed for the participants. Data from the three trials were compared using a two-factor (treatment $\times$ time) repeated-measures ANOVA. A Tukey post-hoc test was applied in the case of a significant $(p<0.05) \mathrm{F}$ ratio to locate the differences with the ANOVA. A one-way ANOVA for repeated measures was used to compare peak power (PP), and mean power (MP) among trials. All statistical analyses were analysed using SPSS for Windows version 15.0 software (SPSS, Chicago, USA). All data are reported as means $+\mathrm{SD}$. Statistical significance was set at alpha $<0.05$.

\section{Results}

Participants' descriptive characteristics are displayed in Table I. There were no significant differences $(p=.18)$ between plasma glucose response at rest and during exercise among the three treatments (CON, 15MPE, and 60MPE) (Fig. 1). However, for the 15-

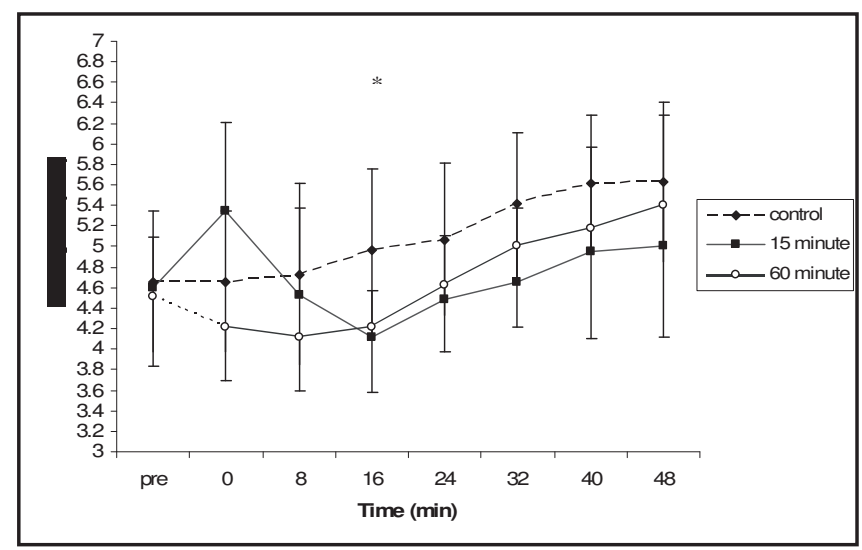

Fig. 1. Plasma glucose concentrations at rest (pre), and during intermittent exercise for control, 15minute, and 60-minute trials. Pre-samples were taken before the subjects ate the bars. Subjects waited 15 minutes or 1 hour. Then another sample was taken at 0 minutes, or at the beginning of the 48-minute exercise protocol. Each of the trials lasted 48 minutes. Plasma glucose concentrations are recorded in $\mathrm{mmol}^{-1} \mathrm{I}^{-1}$. There were no significant differences ( $p>0.05)$ in plasma glucose response between the three trials. However, plasma glucose at 0 , and 16 minutes were significantly different $(p<0.05)$ within the 15MPE feeding trial. Values are means and $S D ; N=10$.

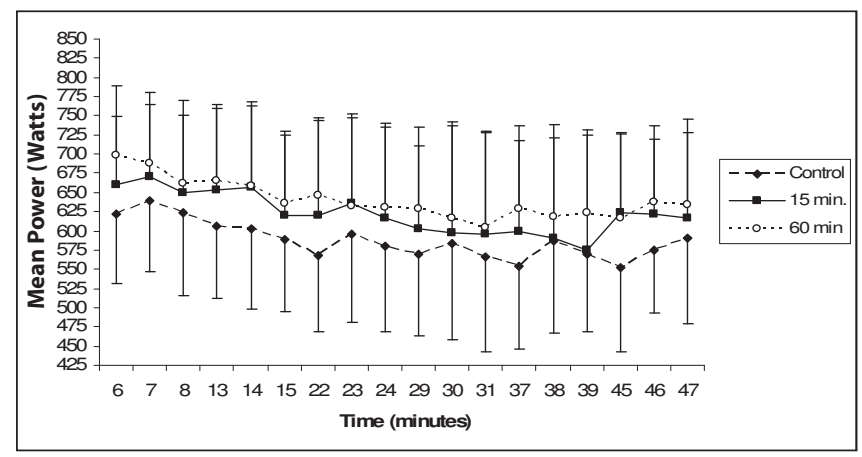

Fig. 2 Mean power (watts) for each $3 \times 1$ - second Wingate sprint session for control, 15MPE and 60MPE trials. Five minutes of cycling at $60 \%$ of $\mathrm{VO}_{2 \max }(\mathrm{ml} / \mathrm{kg} / \mathrm{min})$ took place between each set of $3 \times 10$ Wingate sprints. There were no significant differences $(p>0.05)$ in mean power output between the three trials. Values are means and $S D ; \mathrm{N}=10$. 


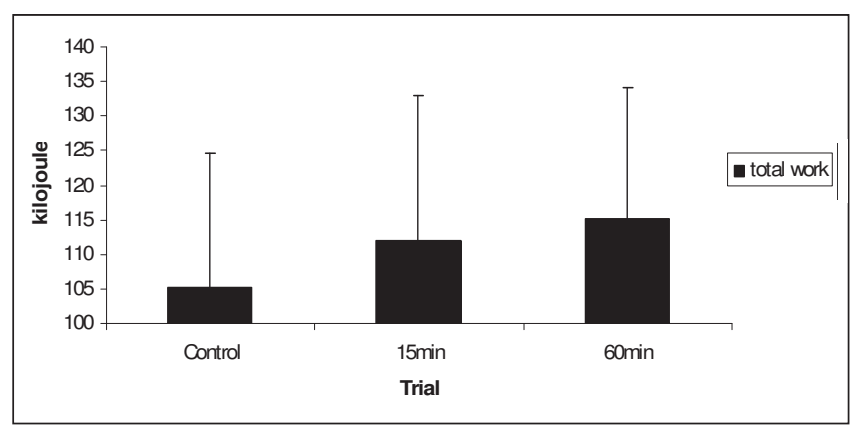

Fig. 3 Total work (kilojoules) during the 18 Wingate sessions for the three trials (control, 15MPE, 60MPE). There were no significant differences ( $p>0.05)$ in total work for the three trials. Values are means and $S D ; N=10$.

minute prior to exercise trial (15MPE), mean plasma glucose was significantly higher $(p<0.05)$ at 0 minutes $\left(5.3 \mathrm{mmol} \cdot \cdot^{-1}\right)$ than at 16 minutes $\left(4.1 \mathrm{mmol} \cdot \mathrm{I}^{-1}\right)$. During the 15MPE trial we observed the expected rise in plasma glucose at the onset of exercise (0 minutes), and then a significant gradual decrease in plasma glucose at 16 minutes during intermittent exercise. There were no significant differences ( $p=0.34$ ) in MP between the three trials (CON 699.2 W; 15MPE 704.6 W; 60MPE 756.1 W) (Fig. 2). No significant differences were seen between the three trials for $\operatorname{PP}(p=0.39)$. Mean total work (joules) was calculated for each trial (mean power $\times$ time) (Fig. 3); no significant difference $(p=0.58)$ was observed between the three trials (CON $105.7 \mathrm{~kJ}$; 15MPE $112.1 \mathrm{~kJ}$; 60MPE $114.9 \mathrm{~kJ}$ ). Furthermore, there was no interaction (treatment $\times$ time) observed for the trials.

\section{Discussion}

The main finding in this study was that the time of ingestion of preexercise nutrition did not affect intermittent cycling performance. The decline in blood glucose during the 15MPE seen between 0 and 16 minutes did not affect intermittent cycling performance. Our findings support other studies that found performance was not affected by the timing of pre-exercise nutrient ingestion. ${ }^{6,8,9,19}$

Most studies have focused on carbohydrate-only fluid ingestion and subsequent results on steady-state cycling performance. ${ }^{12,14}$ However, many athletes may choose to eat a small meal in solid form and not necessarily a liquid carbohydrate beverage as part of their pre-exercise nutritional regimen. ${ }^{20}$ This meal may be a slice of bread with peanut butter, or a piece of fruit and trail mix. The nutritional bar used in this study is more representative (similar consistency and including different percentages of macronutrients and fibre) of these pre-exercise meals than liquids. Based on our findings, the nutrient content of the bar under the current study conditions did not affect intermittent cycling performance. Therefore, the amount of $\mathrm{CHO}$ and protein in the bar used in this study may be eaten in the hour before exercise with no adverse effects on performance.

Total work (joules) for the 18 Wingate tests was not significantly $(p>0.05)$ different between the three trials. However, it may be of practical significance that the 15MPE and 60MPE trials produced more total work ( $5 \% 15 \mathrm{MPE}, 10 \% 60 \mathrm{MPE}$ ) than the CON, suggesting that consuming a meal prior to an event may have some benefits.

Nutrient timing research has primarily focused on endurance performance. The majority of the literature has suggested that endurance performance is not affected ${ }^{8,9,14}$ by the ingestion of carbohy- drate before exercise despite markedly different insulin and glucose concentrations prior to exercise. ${ }^{19}$ Despite the lower $\mathrm{CHO}$ and mixed nutrient composition of the bar used in this study, we observed a similar finding, i.e. that pre-exercise nutrient timing did not impact on high-intensity intermittent exercise performance. However, some studies have found that pre-exercise feedings of glucose caused a negative effect on performance. ${ }^{16,19}$ Foster et al. found a decrease in performance following rebound hypoglycaemia. Investigators have described hypoglycaemia as glucose concentrations less than 3.5 $\mathrm{mmol} \cdot \mathrm{I}^{-1}{ }^{10}$ Although this hypoglycaemic response has been seen consistently in the literature, ${ }^{4,12,14,15}$ it has not been shown to reduce endurance performance. In the present study, rebound hypoglycaemia was defined a priori as plasma glucose concentrations less than $3.5 \mathrm{mmol} \cdot I^{-1}$. No subjects had concentrations lower than $3.7 \mathrm{mmol} \cdot \mathrm{I}^{-1}$ during the three trials. During the 15MPE trial, a rise in blood glucose was observed at the beginning of exercise ( 0 minutes), and then a drop in blood glucose between 0 and 16 minutes occurred $(p<0.05)$. The mean blood glucose levels did not drop below $\left.4.1 \mathrm{mmol} \cdot\right|^{-1}$, and did not seem to affect performance during the Wingate sprints. Because this study did not evoke a hypoglycaemic response we could not evaluate the impact of hypoglycaemia on high-intensity, intermittent exercise performance. Also, no significant differences in RPE verall, leg, or chest were observed among the three trials.

\section{Practical application}

A considerable amount of research has examined nutrient timing prior to an endurance event. However, little research has examined pre-exercise nutrient timing for high-intensity intermittent workouts or competition. The results of this study suggest that nutrient ingestion similar in composition to the feeding used in this study, in the 1 hour prior to high-intensity intermittent activity does not seem to be detrimental to performance. It appears that the use of foods in similar quantity and composition to that tested in this study does not negatively impact performance, and may be consumed as the athlete desires without negative consequences. However, in some sports, such high-intensity sprints may be continued for a longer time period (up to 90 seconds) than that used in this study, and the potential for reactive hypoglycaemic response may be greater. Future research is warranted to examine the effects of pre-exercise nutrient timing on exercise performance with high-intensity bouts of duration longer than 10 seconds.

\section{Conclusion}

The ingestion of a mixed-nutrient energy bar at 15 minutes or 60 minutes before exercise did not affect intermittent cycling performance or blood glucose. In the 15MPE trial we observed a significant rise in blood glucose at 0 minutes and a significant drop in blood glucose between 0 and 16 minutes when compared with the CON and $60 \mathrm{MPE}$ trials. Based on these results, we conclude that the timing and ingestion of a commercially available energy bar that is similar in composition and volume in the hour prior to exercise does not impact on performance.

\section{Acknowledgements}

This study was supported by a grant from USANA, Health Sciences, Inc. 


\section{Just what the doctor ordered.}

\section{REFERENCES}

1. American College of Sports Medicine, American Dietetic Association, and Dietitians of Canada. Nutrition and Athletic Performance. Joint Position Statement of the American Dietetic Association, Dietitians of Canada, and the Medicine and American College of Sports Medicine. Med Sci Sports Exerc 2000; 32(12): 2130-45

2. American College of Sports Medicine. Guidelines for Exercise Testing and Prescription, 6th ed. Philadelphia, $\mathrm{Pa}$ : Lippincott Williams and Wilkins, 2000

3. Borg G. Psychophysical bases of perceived exertion. Med Sci Sports Exerc 1982; 14: 377-81.

4. Chyrssthanopoulos $C$, Hennessy LC. The influence of pre-exercise glucose ingestion on endurance running capacity. Br J Sports Med 1994; 34(3): 235-41.

5. Coleman E. Carbohydrate and exercise. In: Dunford M, ed. A Practice Manual for Professions. Chicago, IL: American Dietetic Association, 2006 : 14-32.

6. Delvin JT, Calles-Escandon J. Effects of pre-exercise snack feeding on endurance cycling exercise. J Appl Physiol 1986; 60: 980-5.

7. Febbraio MA, Chiu A, Angus DJ, Arkinstall MJ, Hawley JA. Effects of carbohydrate ingestion before and during exercise on glucose kinetics and performance. J Appl Physiol 2000; 89: 2220-6.

8. Febbraio MA, Keenan J. Pre-exercise carbohydrate ingestion, glucose kinetics, and muscle glycogen use: effect of glycemic index. J App/ Physiol 2000; 89:1845-51.

9. Febbraio MA, Stewart K. Carbohydrate feedings before prolonged exercise: effect of glycemic index on muscle glycogenolysis and exercise performance. J Appl Physiol 1996; 81: 1115-20.

10. Foster C Costill DL. Effects of pre-exercise feedings on endurance performance. Med Sci Sports 1979; 11: 1-5.

11. Gleeson M, Maughn RJ. Comparison of the effects of pre-exercise feed ings of glucose, glycerol, and placebo on endurance and fuel homeostasis in man. Eur J Appl Physiol 1986; 55: 645-53.

12. Hargreaves M, Hawley JA, Jeukendrup JA. Pre-exercise carbohydrate and fat ingestion: effects on metabolism and performance. J Sports Sci 2004; 22: 31-8.

13. Hargreaves M. Pre-exercise nutritional strategies: effects on metabolism and performance. Can J Appl Physiol 2000; 26: S64-70.

14. Jentjens $R$, Cale $C$, Gutch $C$, Jeukendrup AE. Effects of pre-exercise ingestion of differing amounts of carbohydrate on subsequent metabolism and cycling performance. Eur J Appl Physiol 2003; 88: 444-52.

15. Jentjens $R$, Jeukendrup AE. Prevalence of hypoglycemia following preexercise carbohydrate ingestion is not accompanied by higher insulin sensitivity. Int J Sport Nutr Exerc Metab 2002; 12: 444-59.

16. Keller KR, Schwarzkopf R. Preexercise snacks may decrease exercise performance. Phys Sports Med 1984; 12: 89-91.

17. Kirwan JP, O'Gorman D. A moderate glycemic meal before endurance exercise can enhance performance. J Appl Physiol 1998; 84: 53-59.

18. Maud PJ, Foster C. Physiological Assessment of Human Fitness. Champaign, IL: Human Kinetics, 1995.

19. Moseley L, Lancaster GI. Effects of timing of pre-exercise ingestion of carbohydrate on subsequent metabolism and cycling performance. Eur $J$ Appl Physiol 2003; 88: 453-8.

20. Parcell AC, Drummond MJ, Christopherson ED, Hoyt GL, Cherry JA. Glycemic and insulinemic responses to protein supplements. J Am Diet Assoc 2004; 104: 1800-4.

21. Pollock ML, Schmidt DH, Jackson AS. Measurement of cardiorespiratory fitness and body composition in the clinical setting. Clin Ther 1980; 6: $12-27$.

22. Sherman WM, Peden MC. Carbohydrate feedings 1 hour before exercise improves cycling performance. Am J Clin Nutr 1991; 54: 866-70.

23. Sparks MJ, Selig SS. Pre-exercise carbohydrate ingestion: effect of the glycemic index on endurance performance. Med Sci Sports Exerc 1998; 30: 844-89.

24. Speedy D, Kelly M. The effect of pre-exercise feeding on endurance exercise performance. New Zealand J Sports Med 1998; 26: 34-7.

25. Thomas DE, Brotherhood JR. Carbohydrate feeding before exercise: effect of glycemic index. Int J Sports Med 1991; 12: 180-6.
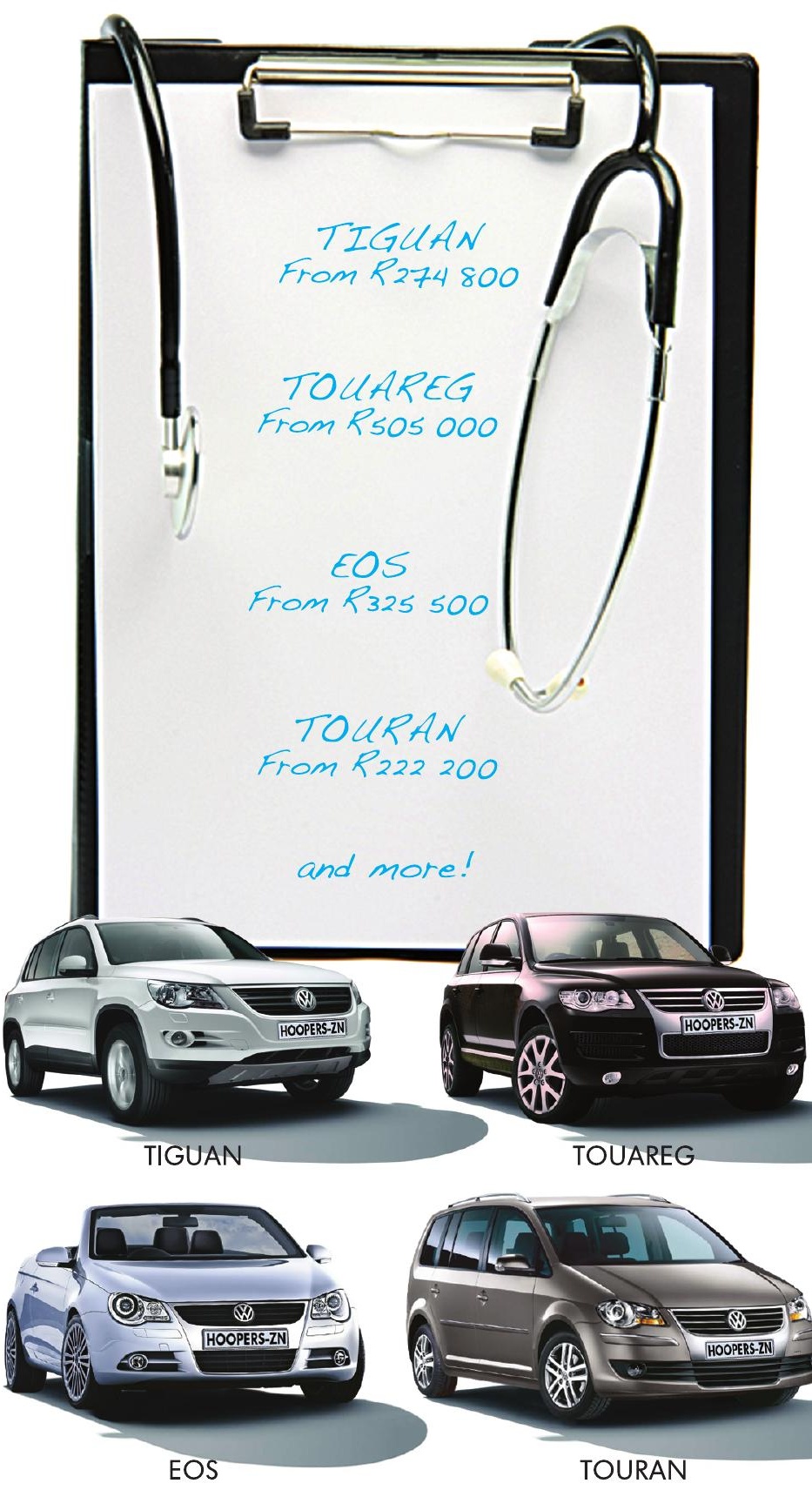

\section{NOW YOU CAN SAVE ON IMPORTED} VOLKSWAGEN MODELS!

\section{Come visit us at HOOPERS}

\& enquire about our special rates!

Safe in-house parking available.

For more details visit www.hoopers.co.za

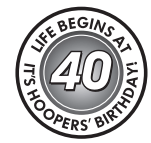

\section{Hoopers}

164 Smith Street, Durban. Tel 0313686868 Ext. 12 